05

\title{
„Изменение“ решеточных свойств и температуры плавления гранецентрированной кубической структуры железа при сжатии
}

\author{
(C) М.Н. Магомедов
}

Институт проблем геотермии Дагестанского научного центра РАН, 367030 Махачкала, Россия

e-mail: mahmag4@mail.ru

(Поступило в Редакцию 28 июня 2016 г.)

Определены все четыре параметра парного межатомного потенциала Ми-Леннард-Джонса и с использованием ранее предложенного метода рассчитаны уравнение состояния $(P)$ и барические зависимости решеточных свойств гранецентрированной кубической структуры железа. Изучены зависимости следующих свойств: температура Дебая, первый, второй и третий параметры Грюнайзена, $B_{T}$ - изотермический модуль сжатия и $B^{\prime}(P), C_{v}$ - изохорная теплоемкость и $C_{v}^{\prime}(P), C_{p}-$ изобарная теплоемкость, $\alpha_{p}-$ коэффициент теплового расширения и $\alpha_{p}^{\prime}(P), \sigma$ - удельная поверхностная энергия и $\sigma^{\prime}(P)$. Расчеты, выполненные вдоль двух изотерм $(1500$ и $3000 \mathrm{~K})$, показали хорошее согласие с экспериментальными данными. Получены аналитические аппроксимации барических зависимостей $B^{\prime}(P), \alpha_{p}(P), C_{p}(P), \sigma^{\prime}(P)$, и показано, что при $P \rightarrow \infty$ функции $B_{T}(P)$ и $\sigma(P)$ изменяются по линейной зависимости, а функции $\alpha_{p}^{\prime}(P)$ и $C_{p}^{\prime}(P)$ стремятся к нулю. Рассчитанная барическая зависимость температуры плавления показала хорошее согласие с экспериментальными данными.

DOI: 10.21883/JTF.2017.04.44315.1954

\section{Введение}

При низких давлениях $(P \cong 0)$ твердое железо может существовать в трех кристаллических модификациях $[1-4]$ :

- при температурах $T \leq 1185 \mathrm{~K}$ стабильна фаза $\alpha$-Fe с объемно-центрированной кубической (ОЦК) структурой: $k_{n}=8, k_{p}=0.6802$, где $k_{n}$ - первое координационное число, $k_{p}$ - коэффициент упаковки структуры;

- при $1185 \mathrm{~K} \leq T \leq 1667 \mathrm{~K}$ стабильна фаза $\gamma$-Fe с гранецентрированной кубической (ГЦК) структурой: $k_{n}=12, k_{p}=0.7405$

— при $1667 \mathrm{~K} \leq T \leq T_{m}=1811 \mathrm{~K}$ стабильна фаза $\delta$-Fe с ОЦК структурой.

Зависимость свойств различных фаз железа от давления изучается уже давно, но до сих пор осталась неясной зависимость решеточных свойств от величины гидростатического (всестороннего) давления $(P)$ вдоль различных изотерм при высоких давлениях (при $P>1000 \mathrm{kbar}=100 \mathrm{GPa})$. Используемые для изучения как уравнения состояния, так и свойств железа при высоких давлениях различные (как численные, так и аналитические) методы содержат в своем формализме подгоночные константы, которые лишены физического смысла, и поэтому предсказать их барическую зависимость вдоль изотермы очень затруднительно. Кроме того, такие методы дают результат только в численно-графическом виде, что делает эти результаты не удобными для дальнейших приложений. Между тем большое количество прикладных задач нуждаются в ясных аналитических зависимостях свойств от давления. В частности, для построения модели Земли необходимы аналитические зависимости свойств различных кристаллических модификаций железа от давления и температуры.

В работах [5-7] были рассчитаны свойства железа с ОЦК структурой. В настоящей работе рассчитаны барические зависимости решеточных свойств $\gamma$-Fe с ГЦК структурой. При этом для расчета уравнения состояния и барической зависимости решеточных свойств использован метод, предложенный в работах [5-7]. Данный метод использует модель кристалла Эйнштейна, приближение „взаимодействия ближайших соседей“ и парный потенциал межатомного взаимодействия Ми-Леннард-Джонса, который имеет вид $[8,9]$

$$
\varphi(r)=\frac{D}{(b-a)}\left[a\left(\frac{r_{0}}{r}\right)^{b}-b\left(\frac{r_{0}}{r}\right)^{a}\right],
$$

где $D$ и $r_{0}$ - глубина и координата минимума потенциала, $b>a \geq 1-$ параметры.

Таким образом, первоначально необходимо самосогласованным образом определить четыре параметра межатомного потенциала (1).

\section{O параметрах межатомного потенциала для ГЦК железа}

Первоначально в [8,9] все четыре параметра потенциала (1) однозначно вычислялись из самосогласованной системы четырех уравнений, в которую входили четыре экспериментально определенных при $T=0 \mathrm{~K}$ и $P=0$ величины. Для ОЦК железа брали следующие значения величин: $V_{0}=7.0494 \mathrm{~cm}^{3} / \mathrm{mol}$ - молярный объем, $L_{0}=413.8 \mathrm{~kJ} / \mathrm{mol}$ - удельная энергия атомизации, $\Theta_{0}=478 \mathrm{~K}$ - температура Дебая и $\gamma_{0}=1.7$ - первый 
параметр Грюнайзена. Таким путем для $\alpha$-Fe в $[8,9]$ был получен следующий набор параметров потенциала (1):

$$
\begin{gathered}
r_{0}=2.4775 \cdot 10^{-10} \mathrm{~m}, \quad D / k_{B}=12576.7 \mathrm{~K}, \\
b=8.26, \quad a=3.58 .
\end{gathered}
$$

Здесь $k_{B}-$ постоянная Больцмана.

Именно эти параметры (2) были использованы в работах $[5,6]$ для изучения поверхностных и диффузионных свойств ОЦК железа при изотермическом сжатии. Но, как было указано в $[9,10]$, величина $\Theta_{0}$ определяется из экспериментальных данных не вполне точно. Так как для железа $k_{B} \Theta_{0} / D \ll 1$, вариация величины $\Theta_{0}$ слабо повлияет на вычисления параметров $D$ и $b$, но существенно изменит величину степени притяжения потенциала $(a)$. Поэтому в [7] значение $a$ для ОЦК железа было скорректировано по величине $\alpha_{p}(P, T)$ - коэффициента теплового расширения при $P=0$ и $T=300 \mathrm{~K}$. Для $\alpha$-Fe эта величина равна [1]: $\alpha_{p}(0,300 \mathrm{~K})=3.6 \cdot 10^{-5} \mathrm{~K}^{-1}$. Подгонка под это значение и дала величину степени притяжения потенциала $(1)$, равную $a(\alpha-\mathrm{Fe})=2.95$. Использование параметров потенциала (2) с новым значением $a(\alpha-\mathrm{Fe})=2.95$ позволило в [7] получить намного лучшее согласие с экспериментом как по уравнению состояния ОЦК железа, так и по барической зависимости различных решеточных свойств.

В настоящей работе для ГЦК фазы железа потенциальные параметры $r_{0}, D$ и $a$ были переопределены с учетом свойств $\gamma$-Fe. Значение $r_{0}-$ координаты минимума потенциала (1) для ГЦК структуры определялось по известной зависимости межатомного расстояния от структуры кристалла [11]: если при полиморфном превращении величина первого координационного числа $k_{n}$ возрастает в последовательности

3 (структура $A 9) \rightarrow 4(A 4) \rightarrow 6(A 5) \rightarrow 8(A 2) \rightarrow 12(A 1)$,

то длина межатомной связи возрастает в следующей последовательности:

$$
1.00 \rightarrow 1.02 \rightarrow 1.09 \rightarrow 1.11 \rightarrow 1.14 \text {. }
$$

Отсюда легко получить $r_{0}(12)=r_{0}(8)(1.14 / 1.11)=$ $=2.5445 \cdot 10^{-10} \mathrm{~m}$.

Так как для железа при переходе от ОЦК к ГЦК структуре значение энергии межатомной связи кристалла перераспределяется от $k_{n}=8$ к связям с $k_{n}=12$, то величина глубины парной межатомной связи для ГЦК железа была переопределена по формуле $D(12)=(8 / 12) D(8)$.

Значение $a(\gamma$-Fe $)$ - степень притяжения потенциала (1) для ГЦК $\gamma$-Fe определяли путем подгонки под величину $\alpha_{p}(0,1200 \mathrm{~K})-$ коэффициента теплового расширения $\gamma$-Fe измеренного при $P=0$ и $T=1200 \mathrm{~K}$ :

$$
\alpha_{p}(0,1200 \mathrm{~K}) /\left[10^{-6} \mathrm{~K}^{-1}\right]=67.2[1]-70.86[2] .
$$

Таким самосогласованным образом, для ГЦК $\gamma$-Fe вместо (2) были получены следующие параметры межатомного потенциала (1):

$$
r_{0}=2.5445 \cdot 10^{-10} \mathrm{~m}, \quad D / k_{B}=8384.467 \mathrm{~K},
$$

$$
b=8.26, \quad a=2.12 .
$$

Исходя из (3), характеристики аналитической модели из [5-7] для ГЦК железа при $V / V_{0}=1$ (т. е. при $P \cong 0$ ) будут равны:

$$
\begin{gathered}
V_{0}=N \pi r_{0}^{3} /\left(6 k_{p}\right)=7.01506 \mathrm{~cm}^{3} / \text { mole, } \\
\rho_{0}=m / V_{0}=7.961 \mathrm{~g} / \mathrm{cm}^{3}, \\
\Theta(1)=313.569 \mathrm{~K}, \quad \gamma(1)=1.70398, \\
q(1)=6.037752 \cdot 10^{-3}, \quad z(1)=1.71597 .
\end{gathered}
$$

Здесь $m$ - масса атома: $m(\mathrm{Fe})=55.847$ a.m.u., а температура Дебая $(\Theta)$, первый $\left(\gamma=-(\partial \ln \Theta / \partial \ln V)_{T}\right)$, второй $\left(q=(\partial \ln \gamma / \partial \ln V)_{T}\right) \quad$ и третий $\left(z=-(\partial \ln q / \partial \ln V)_{T}\right)$ параметры Грюнайзена были рассчитаны по методу, предложенному в $[9,10]$.

Отметим, что при расчетах уравнения состояния и свойств ГЦК железа, так же как и в [7], не учитывались ни вакансии в кристаллической решетке, ни самодиффузия атомов, ибо, как показано в [6], их влияние при сжатии кристалла становится пренебрежимо малым. Здесь, так же как и в [5-7], не учитывается вклад в решеточные параметры электронной подсистемы, ибо потенциал (1) описывает парное взаимодействие нейтральных атомов.

Определив параметры межатомного потенциала (1) для ГЦК железа в виде (3), мы провели расчеты уравнения состояния и решеточных свойств по предложенной в $[6,7]$ сравнительно простой аналитической методике.

\section{2. Результаты расчетов}

На рис. 1 показаны изотермы уравнения состояния ГЦК-Fе: $P(\rho)$, где $\rho=m / V$ - плотность. Линии $1-3-$ наши расчеты с потенциалом (3): точечная линия 1 изотерма $300 \mathrm{~K}$, сплошная линия 2 - изотерма $1500 \mathrm{~K}$,

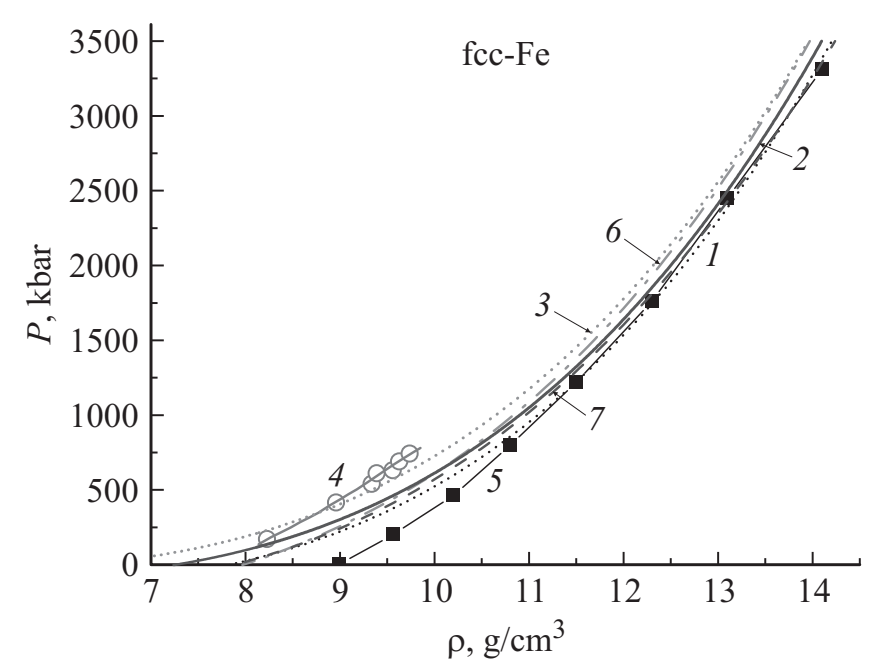

Рис. 1. Изотермы уравнения состояния ГЦК железа, рассчитанные с параметрами (3). Линии $1-3-$ наши расчеты при $T=300,1500,3000 \mathrm{~K}$ соответственно. 
пунктирная линия 3 - изотерма $3000 \mathrm{~K}$. Тонкая сплошная линия 4 - изотерма $298 \mathrm{~K}$, рассчитанная в [12, рис. 10]. Кружки - результаты ударного сжатия $\gamma$-Fe из [12,13]. Тонкая сплошная линия с квадратами $5-$ расчеты упругой изотермы $T=0 \mathrm{~K}$ из [14]. Штрихпунктирная линия 6 - упругая изотерма для ГЦК- $\gamma$-Fe из обзора $[3$, формула (5)]:

$$
P=\frac{\rho_{0}}{n} C_{0}^{2}\left[\left(\frac{\rho}{\rho_{0}}\right)^{n}-1\right],
$$

где для ГЦК- $\gamma$-Fe (измерено до $\sim 0.4 \mathrm{Mbar}): \rho_{0}=$ $=7.98 \mathrm{~g} / \mathrm{cm}^{3}, C_{0}=4.57 \mathrm{~km} / \mathrm{s}, n=4.0$. Отметим, что для гексагональной плотно упакованной фазы (ГПУ- $\varepsilon-\mathrm{Fe})$, измеренной до $\sim 2.5 \mathrm{Mbar}$, получено $\rho_{0}=8.3 \mathrm{~g} / \mathrm{cm}^{3}$, $C_{0}=4.444 \mathrm{~km} / \mathrm{s}, n=4.88$.

Штриховая линия 7 - зависимость, полученная в работе [15, табл. 1] путем аппроксимации экспериментальных данных для ГЦК- $\gamma$-Fe (полученных до $1 \mathrm{Mbar}=100 \mathrm{GPa})$ уравнением Birch-Murnaghan вида

$$
P=1.5 B_{0} \frac{\left(1-x^{2}\right)}{x^{7}}\left[1+\frac{3}{4}\left(B_{0}^{\prime}-4\right) \frac{\left(1-x^{2}\right)}{x^{2}}\right],
$$

где $\quad x=\left(V / V_{0}\right)^{1 / 3}=\left(\rho_{0} / \rho\right)^{1 / 3}, \quad B_{0}=1330 \pm 30 \mathrm{kbar}$, $B_{0}^{\prime}=5$.

Как видно из рис. 1 , наши зависимости $1-3$ хорошо согласуются с зависимостями как из [3] для ГЦК- $\gamma-\mathrm{Fe}$, так и с данными из работ [12-16].

Рассчитав зависимость $P(\rho)$ и зависимость какого-либо свойства $X(\rho)$ вдоль определенной изотермы, можно получить барическую зависимость этого свойства $X(P)$ вдоль этой же изотермы. На рис. 2, $a$ показаны изотермы зависимости для $\Theta(P)$ - температуры Дебая (в $\mathrm{K}$, возрастающие линии, левая шкала) и для $\gamma(P)-$ первого параметра Грюнайзена (спадающие линии, правая шкала). Сплошные линии - изотермы $1500 \mathrm{~K}$, точечные $-T=3000$ К. Квадратами у левой вертикальной оси показаны оценки $\Theta$ при $P=0$ из других работ, которые лежат в интервале $\Theta=335$ [17]-470 K [15]. Оценки для первого параметра Грюнайзена при $P=0$ лежат в области $\gamma=1.3-2.2[3]$.

На рис. 2, $b$ показаны барические зависимости второго $q(P)$ и третьего $z(P)$ параметров Грюнайзена. Оценки других авторов для $q$ лежат в довольно широком интервале: $q=0.69-1.7$ - для ГЦК и ГПУ железа [3], $1.6 \pm 0.6$ - для ГЦК железа [15], 1.2-2.0 - для ГЦК и ГПУ железа [18], $q=(-0.978)-(+0.489)-$ для ГПУ железа [19].

Из рис. 2 видно, что при изобарическом нагреве ГЦК железа функции $\Theta, q$ и $z$ уменьшаются, а величина $\gamma$ возрастает. Отметим, что, как показано в $[3,19]$, известные из работ разных авторов зависимости $\Theta\left(V / V_{0}\right)$ и $\gamma\left(V / V_{0}\right)$ имеют такой широкий разброс, что полученные здесь зависимости $\Theta(P)$ и $\gamma(P)$ хорошо укладываются в эти интервалы. Что касается зависимостей $q(P)$ и $z(P)$, то их оценки никто не проводил, причем в работах других авторов величину $q$ необоснованно считали независящей
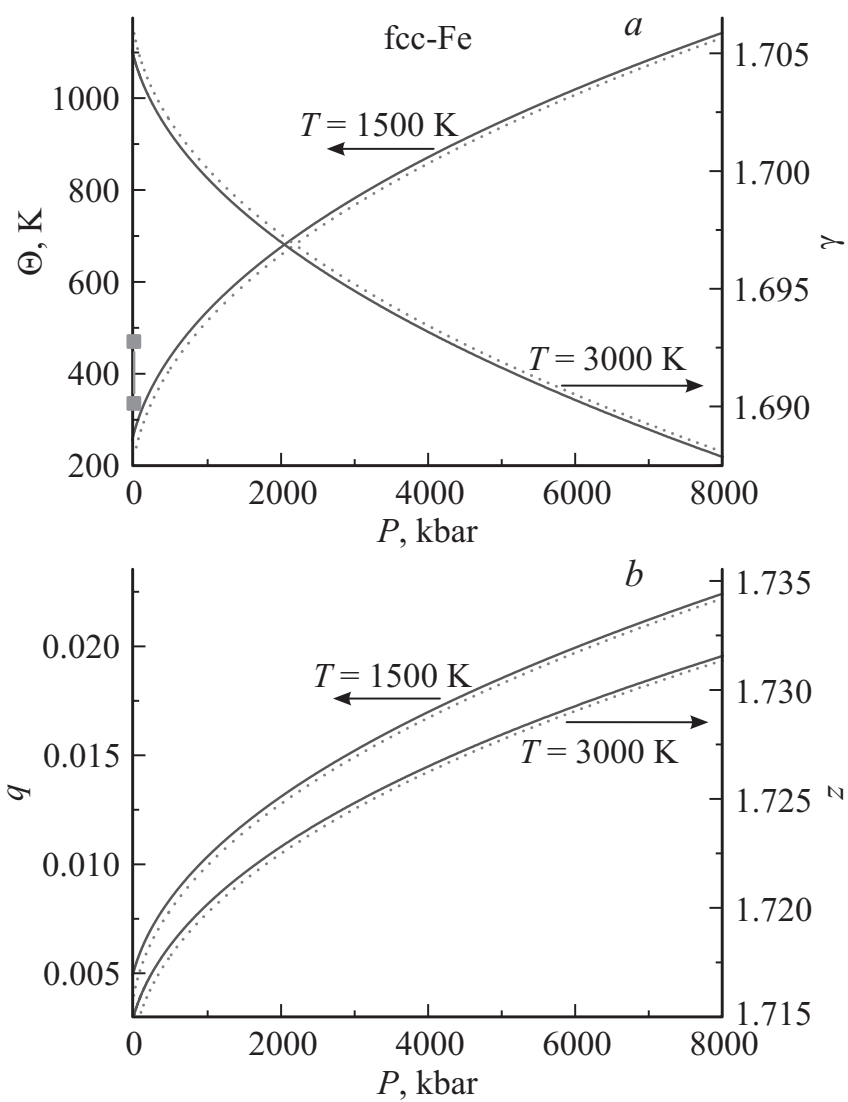

Рис. 2. $a-$ изотермы зависимости $\Theta(P)-$ температуры Дебая (в К, возрастающие линии, левая шкала) и $\gamma(P)-$ первого параметра Грюнайзена (спадающие линии, правая шкала). $b-$ барические зависимости второго $q(P)$ (две верхние линии, левая шкала) и третьего $z(P)$ (две нижние линии, правая шкала) параметров Грюнайзена. Сплошные линии - изотермы $1500 \mathrm{~K}$, точечные $-3000 \mathrm{~K}$.

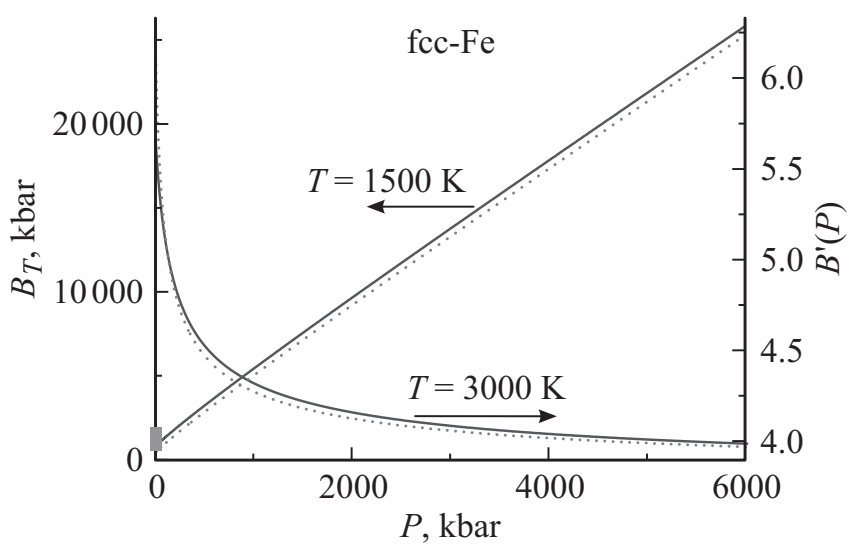

Рис. 3. Изотермы зависимости $B_{T}(P)-$ в kbar, возрастающие линии, левая шкала, и $B^{\prime}(P)-$ спадающие линии, правая шкала. Сплошные линии получены при $1500 \mathrm{~K}$, точечные при $3000 \mathrm{~K}$.

от плотности (или давления), т.е. в этих работах полагали $q=$ const, $z=0$ (см. обзор в $[3,19]$ ). 
На рис. 3 показаны изотермы зависимости $B_{T}(P)=$ $=-V(\partial P / \partial V)_{T}-$ изотермического модуля сжатия (в kbar, возрастающие линии, левая шкала) и $B^{\prime}(P)=$ $=\left(\partial B_{T} / \partial P\right)_{T}-$ производной модуля сжатия по давлению вдоль изотермы (спадающие линии, правая шкала). Сплошные линии получены при $1500 \mathrm{~K}$, точечные при $3000 \mathrm{~K}$. Квадратами у левой вертикальной оси показаны оценки других авторов для ГЦК-Fе при $P=0$ : $B_{T} / \mathrm{kbar}=(889 \pm 51) \quad[16]-(1330 \pm 30) \quad[15]-1550 \quad[3]$. Известные из литературы оценки для производной модуля упругости ГЦК-Fе лежат в интервале: $B^{\prime}(P)=$ $=4.3-(8.9 \pm 0.7)[16]$. Наши данные хорошо вписываются в указанные интервалы.

Из рис. 3 видно, что при низких давлениях из наших расчетов следует $B^{\prime}(1500 \mathrm{~K})<B^{\prime}(3000 \mathrm{~K})$. Но с ростом давления это неравенство изменяется на противоположное. Видно, что с ростом давления функция $B_{T}(P)$ выходит на линейную зависимость, а функция $B^{\prime}(P)$ стремится к постоянной величине.

На рис. 4, $a$ показаны изотермы барических зависимостей для $C_{v} /\left(N k_{B}\right)$ - изохорной (линии 1 и 2 ) и для $C_{p} /\left(N k_{B}\right)-$ изобарной (линии 3 и 4 ) удельных теплоемкостей ГЦК железа. Сплошные линии 1 и 3 получены при $1500 \mathrm{~K}$, пунктирные линии 2 и 4 изотермы $3000 \mathrm{~K}$. Линии 5 и $6-$ результаты расчетов для ГПУ-ع-Fе при $2000 \mathrm{~K}$ из работ [20; 21, рис. 7]. На рис. $4, b$ показаны производные удельной изохорной теплоемкости по давлению (в $10^{-4} / \mathrm{kbar}$ ). Нижняя линия получена при $1500 \mathrm{~K}$, верхняя - при $3000 \mathrm{~K}$. Отметим, что по оценкам из [22] для ОЦК железа при $300 \mathrm{~K}$ для интервала $P=0-100 \mathrm{kbar}$ среднее значение данного параметра равно $C_{v}^{\prime}(P) /\left(N k_{B}\right) \cong$ $\cong-1.54 \cdot 10^{-4} \mathrm{kbar}^{-1}$. В работе [7] для ОЦК железа при $300 \mathrm{~K}$ и $P=0-100 \mathrm{kbar}$ получено $C_{v}^{\prime}(P) /\left(N k_{B}\right) \cong-4.8 \cdot 10^{-4}$ kbar. Для ГПУ-ع-Fе при $2000 \mathrm{~K}$ среднее значение этого параметра равно $C_{v}^{\prime}(P) /\left(N k_{B}\right) \cong-1.2 \cdot 10^{-4} \mathrm{kbar}^{-1}$ (для интевала $P=$ $=500-3500 \mathrm{kbar}) \quad[20], \quad-2.1 \cdot 10^{-4} \mathrm{kbar}^{-1}$ (для $P=$ $=500-2800 \mathrm{kbar})[21$, pис. 7]. Из рис. 4 видно, что при $P \rightarrow \infty$ функции $C_{v}(P)$ и $C_{p}(P)$ стремятся к постоянной величине, а функция $C_{v}^{\prime}(P)$ и разность $C_{p}(P)-C_{v}(P)$ стремятся к нулю. Слабая зависимость $C_{v}$ от давления при $3000 \mathrm{~K}$ есть следствие классического закона Дюлонга-Пти: $C_{v}(T \gg \Theta)=3 N k_{B}$.

На рис. 5, $a$ показаны изотермы зависимости $\alpha_{p}(P)=(\partial \ln V / \partial T)_{P}-$ изобарического коэффициента теплового объемного расширения (в $\left.10^{-5} 1 / \mathrm{K}\right)$ для ГЦК и ГПУ железа. Квадратами около вертикальной оси показаны экспериментальные значения для ГЦК железа при $1200 \mathrm{~K}$ и $P=0$ : $\alpha_{p} /\left[10^{-5} \mathrm{~K}^{-1}\right]=6.75[1]-7.086$ [2]. Две тонкие пунктирные линии на интервале $P=0-3000 \mathrm{kbar}-$ расчетные данные для ГЦК железа из [18]: верхняя кривая для $2000 \mathrm{~K}$, а нижняя - для $1000 \mathrm{~K}$. Две тонкие точечные линии на интервале $180-4000 \mathrm{kbar}$ - расчетные данные для ГПУ-ع-Fe из [19]: верхняя кривая для $9000 \mathrm{~K}$, а нижняя - для $1000 \mathrm{~K}$. Точки и нижняя толстая сплошная линия (для $P=430-1780 \mathrm{kbar})-$ экспериментальные оценки, полученные при $T=1000-5200 \mathrm{~K}$ (см. обзор в $[18,19])$. Наши расчетные изотермы при 1500 и $3000 \mathrm{~K}$ показаны сплошной и пунктирной линиями для всего интервала давлений.

На рис. 5 снизу показаны изотермы барической зависимости для $\alpha_{p}^{\prime}(P)=\left(\partial \alpha_{p} / \partial P\right)_{T}-$ производной $\alpha_{p}$ по давлению (в $\left.10^{-7}(\mathrm{~K} \cdot \mathrm{kbar})^{-1}\right)$, рассчитанные для ГЦК-Fе. Верхняя линия $-T=1500 \mathrm{~K}$, нижняя линия $T=3000 \mathrm{~K}$. Квадратами у вертикальной оси показаны оценки из [22] для ОЦК железа при $T=303-343 \mathrm{~K}$ и $P=0-10$ kbar: $\alpha_{p}^{\prime}(P)=-(4.1-5.05)\left[10^{-7} /(\mathrm{K} \cdot \mathrm{kbar})\right]$. Наши оценки для ОЦК железа при $P=0$ равны [7]: $\alpha_{p}^{\prime}(P) /\left[10^{-7} /(\mathrm{K} \cdot \mathrm{kbar})\right]=-1.2$ для $300 \mathrm{~K},-2.0$ для $1500 \mathrm{~K}$.

Из рис. 5 видно, что при $P \rightarrow \infty$ функция $\alpha_{p}(P)$ стремится к постоянной величине, а функция $\alpha_{p}^{\prime}(P)$ стремится к нулю. Также видно, что при $P \rightarrow \infty$ функция $\alpha_{p}(P)$ перестает зависеть от температуры и изотермы сливаются в одну линию.
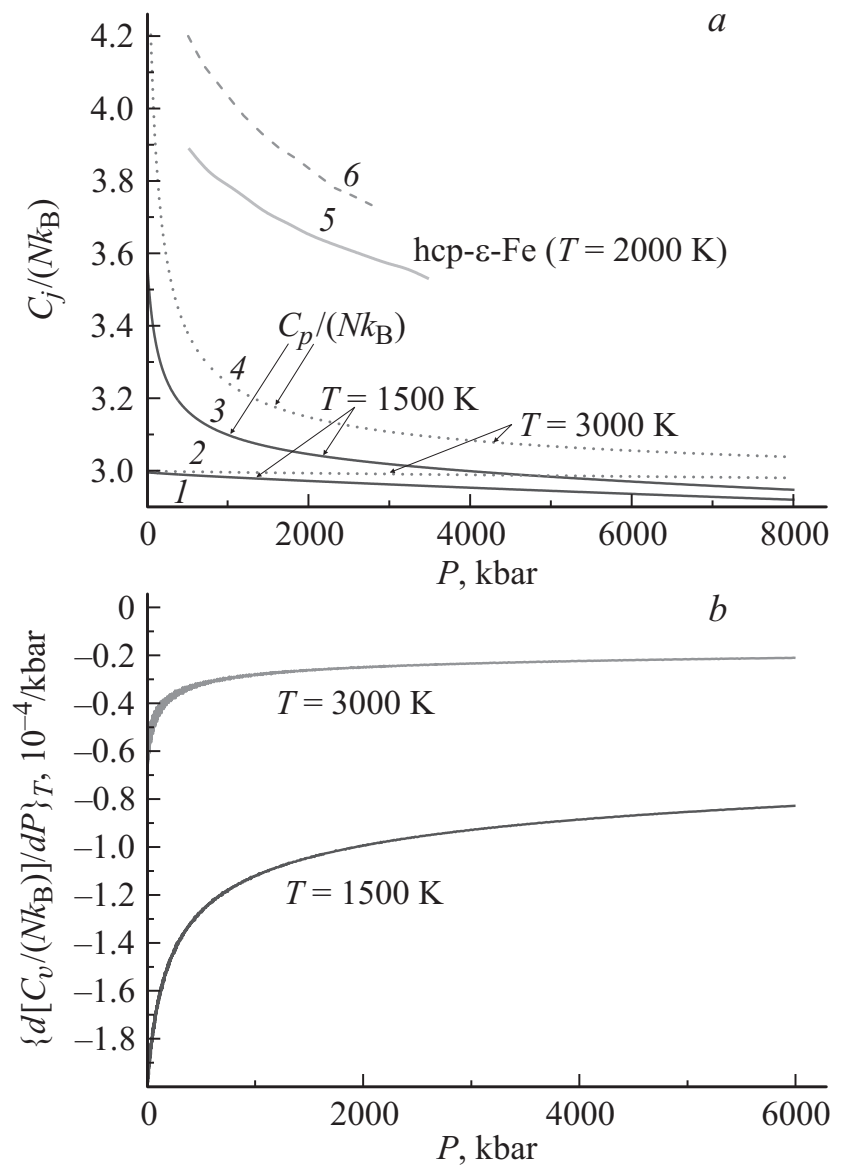

Рис. 4. $a-$ изотермы барических зависимостей для $C_{v} /\left(N k_{B}\right)$ - линии 1 и 2 , и для $C_{p} /\left(N k_{B}\right)$ - линии 3 и 4. Сплошные линии 1 и 3 получены при $1500 \mathrm{~K}$, пунктирные линии 2 и $4-$ при $3000 \mathrm{~K}$. Линии 5 и $6-$ результаты

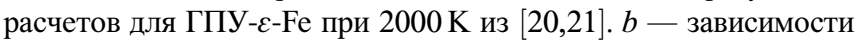
$C_{v}^{\prime}(P) /\left(N k_{B}\right)$ вдоль двух изотерм 1500 и $3000 \mathrm{~K}$. 
Удельная (на единицу площади) поверхностная энергия грани (100) кристалла рассчитывалась по формуле $[9,23]$

$$
\sigma=-\frac{k_{n} D R^{2}}{12 \alpha^{2 / 3} r_{0}^{2}}\left[U(R)+3 H_{w}(R, T)\right],
$$

где введены обозначения $\alpha=\pi /\left(6 k_{p}\right), \quad R=r_{0} / c-$ линейная плотность кристалла, $c=\left[6 k_{p} V /(\pi N)\right]^{1 / 3}-$ расстояние между центрами ближайших атомов,

$$
\begin{gathered}
U(R)=\frac{a R^{b}-b R^{a}}{b-a}, \\
H_{w}(R, T)=\frac{6 \gamma(R)}{(b+2)}\left[\frac{3 k_{B} \Theta(R)}{4 D k_{n}}\right] E_{w}\left(\frac{3 \Theta(R)}{4 T}\right), \\
E_{w}(y)=0.5+\frac{1}{[\exp (y)-1]} .
\end{gathered}
$$

На рис. 6, $а$ показаны изотермы барической зависимости удельной поверхностной энергии $\left(\sigma\right.$ в $\left.10^{-3} \mathrm{~J} / \mathrm{m}^{2}\right)$, а снизу показаны функции $\sigma^{\prime}(P)=(\partial \sigma / \partial P)_{T}$ - производной $\sigma$ по давлению (в $\left.10^{-3} \mathrm{~J} /\left(\mathrm{m}^{2} \cdot \mathrm{kbar}\right)\right)$ для ГЦК железа. Сплошная линия получена при $1500 \mathrm{~K}$, штриховая
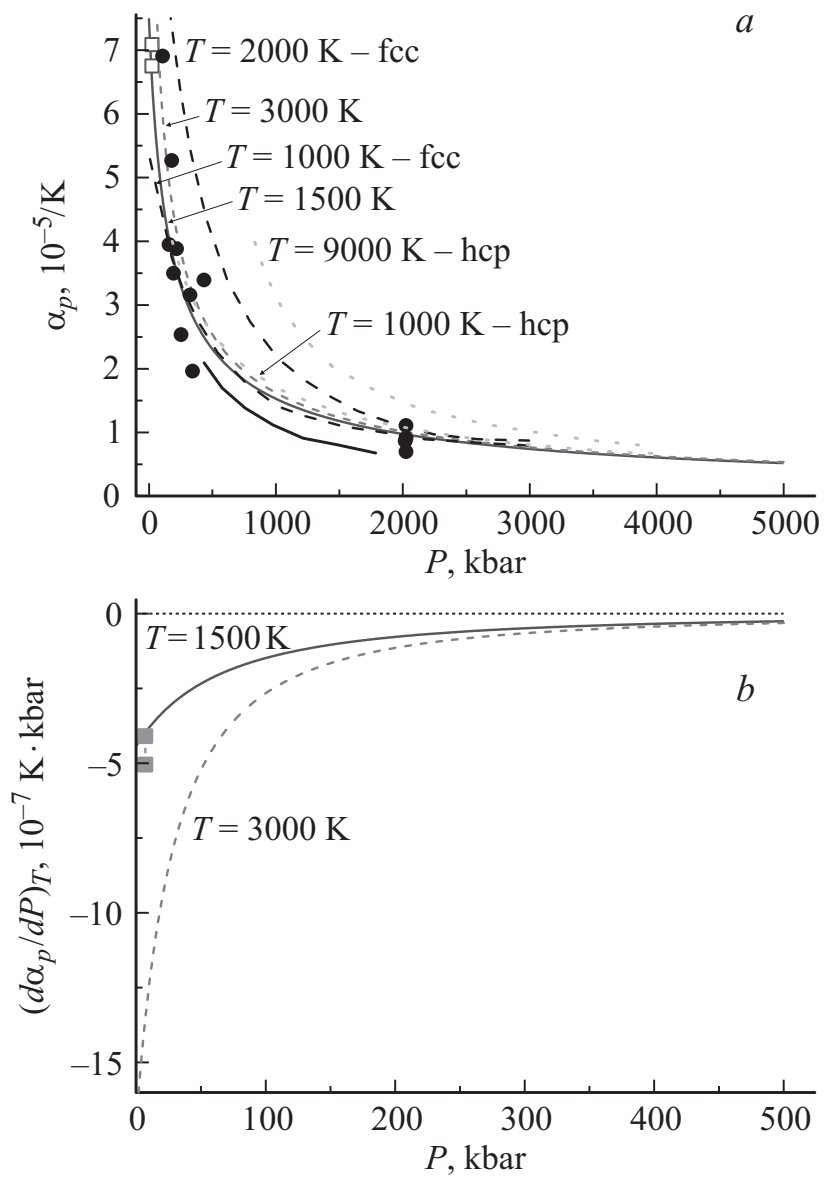

Pис. 5. $a-$ изотермы зависимости коэффициента теплового расширения (в $\left.10^{-5} \mathrm{~K}^{-1}\right)$ для ГЦК и ГПУ железа от давления. $b$ - изотермы зависимости $\alpha_{p}^{\prime}(P)$, рассчитанные для ГЦК железа.
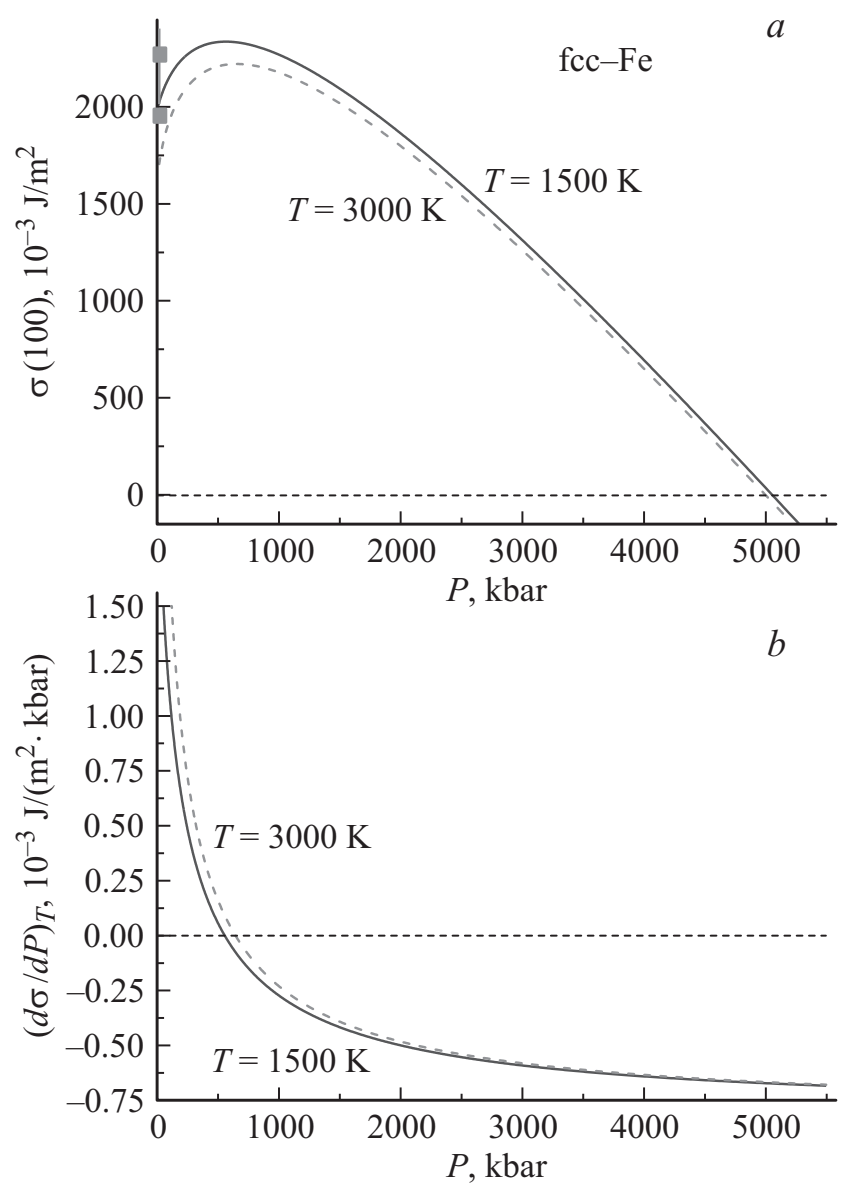

Рис. 6. $a-$ изотермы зависимости $\sigma(P)\left(\right.$ в $\left.10^{-3} \mathrm{~J} / \mathrm{m}^{2}\right), b-$ изотермы для $\sigma^{\prime}(P)$. Квадратами на вертикальной оси показана область оценок $\sigma(100)$ из $[24,25]$.

линия - при $3000 \mathrm{~K}$. Квадратами у вертикальной оси показаны оценки других авторов при $T \leq 300 \mathrm{~K}$ и $P=0$ : $\sigma(100) /\left[10^{-3} \mathrm{~J} / \mathrm{m}^{2}\right]=(1950-2700)[24]-2265[25]$.

Из рис. 6 видно, что выше определенного давления: $P \geq 5046 \mathrm{kbar}\left(V / V_{0} \leq 0.52\right)$ для $1500 \mathrm{~K}$ и $P \geq 4989 \mathrm{kbar}$ $\left(V / V_{0} \leq 0.525\right)$ для $3000 \mathrm{~K}$, величина $\sigma(100)$ становится отрицательной, что должно стимулировать фрагментацию ГЦК кристалла железа на домены с увеличением площади междоменной поверхности.

\section{3. Аппроксимация барических зависимостей}

Выше были представлены в графическом виде барические зависимости решеточных свойств. Но для прикладных задач эти зависимости необходимы в аналитическом виде. Как видно из рис. 3-6, зависимости $B^{\prime}(P), C_{p}(P)$, $\alpha_{p}(P)$ и $\sigma^{\prime}(P)$ на интервале $P=0-4000 \mathrm{kbar}$ хорошо (с коэффициентом достоверности $R_{c o r}>0.998$ ) описываются функцией экспоненциального распада второго 
Коэффициенты аппроксимации (7) для четырех барических зависимостей из рис. $3-6$ и $R_{c o r}-$ коэффициент достоверности аппроксимации данной барической зависимости функцией (7) на интервале $0-4000 \mathrm{kbar}$

\begin{tabular}{|c|c|c|c|c|c|c|}
\hline \multirow{2}{*}{ Функция } & \multicolumn{6}{|c|}{ Коэффициент } \\
\hline & $y_{0}$ & $A_{1}$ & $t_{1}, \mathrm{kbar}$ & $A_{2}$ & $t_{2}, \mathrm{kbar}$ & $R_{\text {cor }}$ \\
\hline & 4.04858 & 0.80614 & 122.83534 & 0.78231 & 959.36543 & 0.99956 \\
\hline$B^{\prime}(P)$ & 4.0558 & 1.16939 & 69.78074 & 0.90118 & 685.42345 & 0.9989 \\
\hline & 0.672073 & 3.47825 & 112.96816 & 2.95072 & 821.11655 & 0.99955 \\
\hline$\alpha_{p}(P) /\left[10^{-5} / \mathrm{K}\right]$ & 0.832673 & 6.83283 & 61.02518 & 4.54639 & 538.91133 & 0.99895 \\
\hline & 3.00125 & 0.2864 & 122.98692 & 0.24956 & 1092.36868 & 0.9994 \\
\hline$C_{p} /\left(N k_{B}\right)$ & 3.11677 & 1.05802 & 61.79922 & 0.69745 & 556.43107 & 0.9989 \\
\hline & -0.61309 & 1.47225 & 111.06562 & 1.26112 & 774.20283 & 0.99959 \\
\hline$\sigma^{\prime}(P) /\left[10^{-3} \mathrm{~J} /\left(\mathrm{m}^{2} \cdot \mathrm{kbar}\right)\right]$ & -0.55118 & 2.89187 & 60.68517 & 1.97104 & 518.6625 & 0.99903 \\
\hline
\end{tabular}

Примечание. В первой строке коэффициенты для изотермы $1500 \mathrm{~K}$, во второй строке - для $3000 \mathrm{~K}$.

порядка (где $P$ в kbar):

$$
y(P)=y_{0}+A_{1} \exp \left(-\frac{P}{t_{1}}\right)+A_{2} \exp \left(-\frac{P}{t_{2}}\right) .
$$

Поэтому мы подогнали полученные в графическом виде барические зависимости для указанных свойств под функцию (7). Данная функция удобна тем, что из нее путем дифференцирования или интегрирования легко получить барические зависимости для других свойств, например для функций: $B_{T}(P), C_{p}^{\prime}(P), \alpha_{p}^{\prime}(P), \sigma(P)$ и $\alpha_{p} B_{T}=(\partial P / \partial T)_{V}$.

В таблице представлены значения коэффициентов функции (7) для зависимостей $B^{\prime}(P), C_{p}(P) /\left(N k_{B}\right)$, $\alpha_{p}(P)$ и $\sigma^{\prime}(P)$. Из (7) видно, что при $P \rightarrow \infty$ функции $B^{\prime}(P), C_{p}(P) /\left(N k_{B}\right), \alpha_{p}(P)$ и $\sigma^{\prime}(P)$ стремятся к постоянным величинам, равным $y_{0}$ из таблицы. Это означает, что при $P \rightarrow \infty$ функции $B_{T}(P)$, и $\sigma(P)$ изменяются по линейной зависимости, а функции $C_{p}^{\prime}(P)$ и $\alpha_{p}^{\prime}(P)$ стремятся к нулю.

\section{4. Барическая зависимость температуры плавления ГЦК железа}

Для изучения зависимости температуры плавления $\left(T_{m}\right)$ от давления используем формулу Линдеманна. Тогда при $T_{m} \gg \Theta$ для зависимости $T_{m}$ от относительного объема $\left(V / V_{0}\right)$ можно получить выражение вида [26]

$$
T_{m}=T_{m}(0)\left(\frac{V}{V_{0}}\right)^{2 / 3}\left[\frac{\Theta\left(V / V_{0}\right)}{\Theta(1)}\right]^{2}=T_{m}(0)\left(\frac{V}{V_{0}}\right)^{-b / 3}
$$

где $T_{m}(0)-$ температура плавления при $P=0$, $\Theta(1)=\Theta\left(V / V_{0}=1\right)$.

Из экспериментально определенного выражения (4) легко получить

$$
\frac{V}{V_{0}}=\left(1+\frac{n P}{\rho_{0} C_{0}^{2}}\right)^{-1 / n}
$$

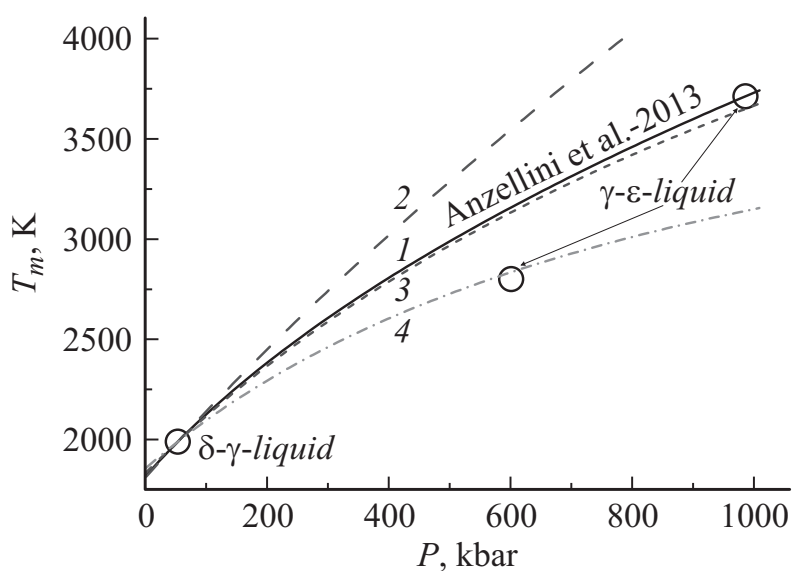

Рис. 7. Зависимость температуры плавления ГЦК железа от давления. Сплошная линия 1 - экспериментальная зависимость (11) из [4]. Кружки указывают положение тройных точек на линии плавления. Верхняя штриховая линия $2-$ зависимость (12) при $\overline{\alpha_{p}}=0$, средняя точечная линия $3-$ при $\overline{\alpha_{p}}=3 \cdot 10^{-5} \mathrm{~K}^{-1}$, нижняя штрихпунктирная линия $4-$ при $\overline{\alpha_{p}}=7 \cdot 10^{-5} \mathrm{~K}^{-1}$.

Учтя, что для железа тройная точка $\delta$ - $\gamma$-liquid имеет хорошо определенные координаты $[3,4]: P_{t 1}=52 \mathrm{kbar}$, $T_{m}\left(P_{t 1}\right)=1991 \mathrm{~K}$, из формул $(8)$ и (9) получим

$$
T_{m}(P)=T_{m}\left(P_{t 1}\right)\left[F\left(P-P_{t 1}\right)\right]^{b / 3},
$$

где

$$
F\left(P-P_{t 1}\right)=\left[1+\frac{n\left(P-P_{t 1}\right)}{\rho_{0} C_{0}^{2}}\right]^{1 / n} .
$$

На рис. 7 показаны барические зависимости для температуры плавления ГЦК железа. Сплошная линия 1 экспериментальная зависимость из [4] (давление в kbar):

$$
T_{m}(P)=T_{m}\left(P_{t 1}\right)\left[1+\frac{\left(P-P_{t 1}\right)}{273.9}\right]^{1 / 2.38} .
$$

Верхняя штриховая линия 2 - расчет по формуле (10). 
Кружками на рис. 7 показаны тройные точки на линии плавления железа:

$$
\begin{gathered}
1-\delta \text { - } \gamma \text {-liquid }[3,4]: P_{t 1}=52 \mathrm{kbar}, T_{m}\left(P_{t 1}\right)=1991 \mathrm{~K}, \\
2-\gamma \text { - } \varepsilon \text {-liquid из [3]: } P_{t 2}=600 \pm 50 \mathrm{kbar}, \\
T_{m}\left(P_{t 2}\right)=2800 \pm 200 \mathrm{~K}, \\
3-\gamma \text { - } \varepsilon \text {-liquid из [4]: } P_{t 2}=985 \pm 50 \mathrm{kbar}, \\
T_{m}\left(P_{t 2}\right)=3712 \pm 200 \mathrm{~K} .
\end{gathered}
$$

Отметим, что в литературе до сих пор нет единого мнения о координатах тройной точки $\gamma$ - $\varepsilon$-liquid-Fe, например, в [12] брали: $P_{t 2}=810 \mathrm{kbar}, T_{m}\left(P_{t 2}\right)=2800 \mathrm{~K}$.

Из рис. 7 видно, что зависимость $T_{m}(P)$ из (10) проходит выше экспериментальной зависимости из [4], что указывает на приближенность формулы (9): в ней не учитывается рост объема кристалла с ростом $T_{m}(P)$. При учете увеличения объема кристалла с ростом $T_{m}(P)$ вместо формулы (10) получим интегральное уравнение вида

$$
\begin{aligned}
T_{m}(P)= & T_{m}\left(P_{t 1}\right)\left[F\left(P-P_{t 1}\right)\right]^{b / 3} \\
& \times\left\{1+F\left(P-P_{t 1}\right) \int_{T_{m}\left(P_{t 1}\right)}^{T_{m}(P)} \alpha_{p}(T, P) d T\right\}^{-b / 3} .
\end{aligned}
$$

Полагая, что второе слагаемое в фигурных скобках много меньше единицы, и используя теорему о среднем интегрального исчисления, можно получить выражение

$$
\begin{aligned}
& T_{m}(P)=T_{m}\left(P_{t 1}\right) \\
& \times \frac{\left[F\left(P-P_{t 1}\right)\right]^{b / 3}\left\{1+\left(\frac{b}{3}\right) \overline{\alpha_{p}} T_{m}\left(P_{t 1}\right) F\left(P-P_{t 1}\right)\right\}}{\left\{1+\left(\frac{b}{3}\right) \overline{\alpha_{p}} T_{m}\left(P_{t 1}\right)\left[F\left(P-P_{t 1}\right)\right]^{1+(b / 3)}\right\}},
\end{aligned}
$$

где $\overline{\alpha_{p}}$ - среднее значение коэффициента теплового расширения $\alpha_{p}(T, P)$ на интервале от $T_{m}\left(P_{t 1}\right)$ до $T_{m}(P)$. Легко видеть, что при $\overline{\alpha_{p}}=0$ формула (12) переходит в (10).

На рис. 7 кривой 3 показана зависимость (12) при $\overline{\alpha_{p}}=3 \cdot 10^{-5} \mathrm{~K}^{-1}$, а нижней штрихпунктирной линией 4 - при $\overline{\alpha_{p}}=7 \cdot 10^{-5} \mathrm{~K}^{-1}$. Таким образом, формула (12) позволяет описать зависимость $T_{m}(P)$ для ГЦК железа.

\section{Заключение}

1. Используя метод из $[6,7]$ и корректно определенные параметры межатомного потенциала (1), можно рассчитать изотермы уравнения состояния и барические зависимости решеточных свойств как для ОЦК, так и для ГЦК железа.

2. Получены барические зависимости следующих свойств ГЦК железа: температура Дебая, первый, второй и третий параметры Грюнайзена, $B_{T}$ - изотермический модуль сжатия и $B^{\prime}(P), C_{v}-$ изохорная теплоемкость и $C_{v}^{\prime}(P), C_{p}$ - изобарная теплоемкость, $\alpha_{p}-$ коэффициент теплового расширения и $\alpha_{p}^{\prime}(P), \sigma$ - удельная поверхностная энергия и $\sigma^{\prime}(P)$. Расчеты, выполненные вдоль изотерм 1500 и $3000 \mathrm{~K}$, показали хорошее согласие с экспериментальными данными.

3. Получены сравнительно простые аналитические аппроксимации функций $B^{\prime}(P), C_{p}(P), \alpha_{p}(P)$ и $\sigma^{\prime}(P)$, из которых видно, что при $P \rightarrow \infty$ функция $B_{T}(P)$ возрастает, а $\sigma(P)$ убывает по линейной зависимости. Функции $C_{p}^{\prime}(P)$ и $\alpha_{p}^{\prime}(P)$ для ГЦК железа при $P \rightarrow \infty$ стремятся к нулю из отрицательной области значений.

4. Получено аналитическое выражение для зависимости температуры плавления ГЦК железа от давления. Показано, что для корректного описания функции $T_{m}(P)$ необходимо учитывать увеличение объема кристалла с ростом величины $T_{m}(P)$.

Автор выражает благодарность Э.Н. Ахмедову, Н.Ш. Газановой, З.М.Сурхаевой и М.М. Гаджиевой за плодотворные дискуссии и помощь в работе.

Работа выполнена при финансовой поддержке РФФИ в рамках научного проекта № 16-03-00041_а и Программы Президиума РАН (программа № I.11П(1)).

\section{Список литературы}

[1] Новикова С.И. Тепловое расширение твердых тел. М.: Наука. 1974. 294 с.

[2] Liu Y.C., Sommer F., Mittemeijer E.F. // Thermochimica Acta. 2004. Vol. 413. P. 215-225.

[3] Фунтиков А.И. // Теплофизика высоких температур. 2003. Т. 41. Вып. 6. С. 954-969.

[4] Anzellini S., Dewaele A., Mezouar M., Loubeyre P., Morard G. // Science. 2013. Vol. 340. N 6131. P. 464-466.

[5] Магомедов М.Н. // Поверхность. Рентген., синхротр. и нейтрон. исслед. 2012. Вып. 5. С. 59-71.

[6] Магомедов М.Н. // ЖТФ. 2013. Т. 83. Вып. 3. С. 71-78.

[7] Магомедов М.Н. // ЖТФ. 2015. Т. 85. Вып. 11. С. 48-54.

[8] Магомедов М.Н. // Теплофизика высоких температур. 2006. Т. 44. Вып. 4. С. 518-533.

[9] Магомедов М.Н. Изучение межатомного взаимодействия, образования вакансий и самодиффузии в кристаллах. М.: Физматлит. 2010. 544 c.

[10] Магомедов М.Н. // ФТТ. 2003. Т. 45. Вып. 1. С. 33-36.

[11] Бацанов С.С. Структурная химия. Факты и зависимости. М.: Диалог-МГУ. 2000. 292 с.

[12] Медведев А.Б. Физика горения и взрыва. 2014. Т. 50. Вып. 5. С. 91-108

[13] Chen G.Q., Ahrens T.J. // High-Pressure Materials Research. Warrendale, PA, 1998. P. 41-61 (Mat. Res. Soc. Symp. Proc. 499).

[14] Asker C., Kargen U., Dubrovinsky L., Abrikosov I. // Earth Planet. Sci. Lett. 2010. Vol. 293. N 1-2. P. 130-134.

[15] Campbell A., Danielson L., Righter K., Seagle C.T., Wang Y., Prakapenka V. // Earth Planet. Sci. Lett. 2009. Vol. 286. P. 556-564. 
[16] Nishihara Y., Nakajima Y., Akashi A., Tsujino N., Takahashi E., Funakoshi K., Higo Y. // American Mineralogist. 2012. Vol. 97. P. 1417-1420.

[17] Коноплин Н.А. Термический, барический и концентрационный полиморфизм железа / Автореф. дисс. канд. физ.мат. наук. Москва: Изд-во МИЭТ. 2006. 28 с.

[18] Wasserman E., Stixrude L., Cohen R.E. // Phys. Rev. B. 1996. Vol. 53. N 13. P. 8296-8309.

[19] Belonoshko A.B. // Condens. Matter Physics. 2010. Vol. 13. N 2. P. 23605 (11).

[20] Stixrude L., Wasserman E., Cohen R.E. // J. Geophys. Research. 1997. Vol. 102. N B11. P. 24729-24739.

[21] Vocadlo L., Alfe D., Gillan M.J., Price G.D. // Phys. Earth Planet. Interiors. 2003. Vol. 140. N 1-3. P. 101-125.

[22] Гайваронский A.T. Термодинамические свойства изостатически сжатых поликристаллических металлов. М.: Наука, 1991. $112 \mathrm{c}$

[23] Магомедов М.Н. // ФТТ. 2004. Т. 46. Вып. 5. С. 924-937.

[24] Kumikov V.K., Khokonov Kh.B. // J. Appl. Phys. 1983. Vol. 54. N 3. P. $1346-1350$.

[25] Chamati H., Papanicolaou N.I., Mishin Y., Papaconstantopoulos D.A. // Surf. Sci. 2006. Vol. 600. N 9. P. 1793-1803.

[26] Магомедов М.Н. // ЖТФ. 2008. Т. 78. Вып. 8. С. 93-100. 